\title{
Functional Conducting Polymers in the Application of SPR Biosensors
}

\author{
Rapiphun Janmanee, ${ }^{1,2}$ Sopis Chuekachang, ${ }^{1,2}$ Saengrawee Sriwichai, ${ }^{1}$ \\ Akira Baba, ${ }^{2}$ and Sukon Phanichphant ${ }^{3}$ \\ ${ }^{1}$ Department of Chemistry, Faculty of Science, Chiang Mai University, Chiang Mai 50200, Thailand \\ ${ }^{2}$ Center for Transdisciplinary Research, Graduate School of Science and Technology, Niigata University, Niigata 950-2181, Japan \\ ${ }^{3}$ Materials Science Research Center, Faculty of Science, Chiang Mai University, Chiang Mai 50200, Thailand
}

Correspondence should be addressed to Sukon Phanichphant, sphanichphant@yahoo.com

Received 20 February 2012; Accepted 21 May 2012

Academic Editor: Carlos R. Cabrera

Copyright (๑) 2012 Rapiphun Janmanee et al. This is an open access article distributed under the Creative Commons Attribution License, which permits unrestricted use, distribution, and reproduction in any medium, provided the original work is properly cited.

In recent years, conducting polymers have emerged as one of the most promising transducers for both chemical, sensors and biosensors owing to their unique electrical, electrochemical and optical properties that can be used to convert chemical information or biointeractions into electrical or optical signals, which can easily be detected by modern techniques. Different approaches to the application of conducting polymers in chemo- or biosensing applications have been extensively studied. In order to enhance the application of conducting polymers into the area of biosensors, one approach is to introduce functional groups, including carboxylic acid, amine, sulfonate, or thiol groups, into the conducting polymer chain and to form a so-called "self-doped" or by doping with negatively charged polyelectrolytes. The functional conducting polymers have been successfully utilized to immobilize enzymes for construction of biosensors. Recently, the combination of SPR and electrochemical, known as electrochemical-surface plasmon resonance (EC-SPR), spectroscopy, has been used for in situ investigation of optical and electrical properties of conducting polymer films. Moreover, EC-SPR spectroscopy has been applied for monitoring the interaction between biomolecules and electropolymerized conjugated polymer films in biosensor and immunosensor applications. In this paper, recent development and applications on EC-SPR in biosensors will be reviewed.

\section{Introduction}

Conducting polymers $(\mathrm{CPs})$ are materials discovered over 20 years ago, which have attracted considerable attention for their electronic conducting properties, optical properties, and chemical and biochemical properties [1-8]. These unique properties of CPs have been used in wide range of applications including battery technology, photovoltaic devices, light emitting diodes, electrochromic displays, and more recently in biological application [9-11]. Typical examples of conducting polymers such as polyaniline, poly $(3,4-$ ethylenedioxythiophene), polypyrrole, and their derivatives have been widely studied $[12,13]$. In particular for biosensor application, conducting polymers based on polypyrrole (PPy) have been extensively investigated due to their good electrical conductivity, environmental stability to air and water, and ease of synthesis through electrochemical and chemical routes $[4,14]$. Several techniques coupled to electrochemistry have been developed to study the properties of polymer films such as quartz crystal microbalance (QCM) [15], Fourier transform-infrared (FT-IR) spectroscopy [16], electron spin resonance (ESR) [17], and surface plasmon resonance (SPR) spectroscopy $[18,19]$. SPR spectroscopy is the technique for monitoring the change of the refractive index at a solid-liquid interface, which has been widely used for the characterization and study of polymer films, interfaces, and kinetic processes at surfaces [20-23]. Recently, the combination of SPR and electrochemical, named electrochemicalsurface plasmon resonance (EC-SPR) spectroscopy, have been used for in situ investigation of optical and electrical properties of conducting polymer films [24-26]. Moreover, EC-SPR spectroscopy has been applied for monitoring 
the interaction between biomolecules and electropolymerized conjugated polymer films in biosensor and immunosensor applications [27-30]. In this paper, recent development and applications on EC-SPR in biosensors will be reviewed.

\section{Electrochemical SPR for Monitoring of Conducting Polymer Thin Films}

In 2003, the properties of poly(3,4-ethylenedioxythiophene) (PEDOT) ultrathin films investigated by the combination of surface plasmon resonance (SPR) and surface plasmon enhanced photoluminescence spectroscopy (SPPL) with electrochemical techniques, known as EC-SPPL, were reported by Baba and Knoll [21]. The electrochromic properties and the detecting of photoluminescence in PEDOT ultrathin films were observed. The photoluminescence of PEDOT was observed when the polymer was dedoped under the applied potential. The photoluminescence intensity was controlled by the potential and dependent on the angular position in an SPR reflectivity experiment. The SPR characterization of the PEDOT film is corresponding with the PEDOT bulk electrochromic properties obtained from UV-vis-NIR spectra. The EC-SPPL method can be acted as a sensitive tool for detection the photoluminescence from a conjugated polymer film and could have potentials for sensors and optoelectronic devices applications.

An electroactivity of polyaniline (PANI) films in neutral $\mathrm{pH}$ condition and their electrocatalyzed oxidation of $\beta$-nicotinamide adenine dinucleotide (NADH) were also reported by Tian et al. [23]. Self-assembled PAIN multilayer films by forming with poly(anion) such as sulfonated polyaniline (SPANI), poly(acrylic acid) (PAA), poly(vinyl sulfonate) (PVS), and poly(styrene sulfonate) (PSS) were prepared using layer-by-layer (LBL) method. The combination of EC-SPR and quartz crystal microbalance (QCM) techniques was used to monitor the electrochemical behavior and catalytic ability for the oxidation of $\beta$-nicotinamide adenine dinucleotide (NADH) in neutral solution of PANI multilayer films. The self-assembled PANI multilayer films prepared by LBL method showed very good stability, reversible, and electroactive in neutral solution. Moreover, the results of the electrocatalytic activity of PANI multilayer films indicated that the PAIN copolymers can electrocatalyze the oxidation of $\mathrm{NADH}$ in neutral solution but their potential to electrocatalyze NADH oxidation was quite different under the same condition. The catalytic ability of PANI/SPANI is better than the other assemblies under the same conditions due to both PANI and SPANI monolayers of PANI/SPANI system being electroactive, while for the other systems only the PANI layer is electroactive.

The investigation of the formation and effects of doping/dedoping processes on the optical properties associated with ultrathin polypyrrole (PPy) film using EC-SPR was studied by Damos et al. in 2006 [4]. The electropolymerization of pyrrole was performed by potentiostatic, potentiodynamic, and galvanostatic methods. The in situ EC-SPR was monitored by the nanometric films of PPy. The results showed the electropolymerization mode was essential to the stability of polymer and the reversibility of its optical properties during the doping and dedoping processes. The changes in the electrochemical and optical properties of the thin polypyrrole films produced the changes of the SPR angle position due to the changes in the values of the real and imaginary parts of the dielectric constant for PPy films during doping/dedoping processes. The results from the QCM measurement to monitor the correlation between doping/ dedoping processes and the changes in the real and imaginary parts of the dielectric constant of the polypyrrole film were consistent with the results from EC-SPR technique.

In 2009, an electrochemical surface plasmon excitation and emission light properties in poly(3-hexylthiophene) (P3HT) thin films were investigated by Kato et al. [22]. The electrochemical change of P3HT thin films was in situ measured by the attenuated total reflection (ATR) and emission light properties utilizing surface plasmon (SP) excitations. The ATR and SP emission light properties were observed for the doping-dedoping states of P3HT thin film. The ATR and SP emission light properties were remarkably changed with the electrochemical doping and dedoping. The SP emission light also decreased by decreasing the molecular luminescence of P3HT by doping. For the dedopedstate P3HT thin film, SP emission light also increased by increasing the molecular luminescence. The SP emission light excited by molecular luminescence can be controlled by controlling the doping-dedoping states.

\section{SPR Biosensors Based on Functional Conducting Polymers}

Conducting polymers in the area of biosensors have attracted much attraction. Biosensors based on conducting polymers were used to detect the biomolecules including glucose, hormones, neurotransmitters, antibodies, and antigens [ 1 , 20,31-34]. Immunosensors are biosensors based on specific antigen-antibody interactions and in which the transducer detects either directly or indirectly the immunochemical reactions [35-37]. Polypyrrole and its derivative were studied for the biosensor application $[26,34]$. The EC-SPR spectroscopy can be applied not only in monitoring the electropolymerization of polymer films, but also in illustrating the properties of immunosensors [12, 38, 39].

In 2004, the label-free immunosensor for the detection of a rabbit IgG antigen by an antirabbit IgG antibody at the surface of polypyrrole (PPy) membrane was reported by Gooding et al. [13]. The investigation of the electrochemistry on a glassy carbon electrode, modified with antibody entrapped in an electrodeposited polypyrrole (PPy) membrane, was demonstrated in a flow injection apparatus (FIA). The measured signals were aroused by a change in ion flux into and out of the conducting polymer membrane when the antigen interacted with the antibody-modified polymer. The most sensitivity of the label-free immunosensors was shown by a change of the cyclic voltammograms at $0.4 \mathrm{~V}$ when the antigen was bound. The concentration of the antibody immobilized throughout the polymer membrane plays an important role for altering the electrode stability and has the lowest detectable concentration, but had little effect on the concentration range over which immunosensor operated. 
The reversibility is a consequence of the pulsing of the electrochemical potential on the time scale of $200 \mathrm{~ms}$. This time is insufficient to allow the secondary bonding forces (hydrogen bonding and hydrophobic force), which would render the binding effectively irreversible, from becoming established.

SPR immunosensor for detection of insulin based on oligo(ethyleneglycol)-dithiocarboxylic acid (OEG-DCA) monolayer was studied by Gobi et al. in 2007 [29]. The SPR chip was prepared by a heterobifunctional oligo(ethyleneglycol)-dithiocarboxylic acid derivative (OEG-DCA) containing dithiol and carboxyl end groups to functionalize the thin Au film. Insulin was covalently bound to the Authiolate monolayer of OEG-DCA for activating the sensor surface to immunoaffinity interactions. The sensor chip was monitored in real time for detection of insulin at various concentrations. The lowest detectable concentration of insulin was $1 \mathrm{ng} / \mathrm{mL}$ with a response time of less than $5 \mathrm{~min}$ and the determination range covered a wide concentration range of 1-300 ng/mL. The developed OEG-monolayer-based sensor chip provided exceptional sensor performance characteristics, reproducibility, stability, and high resistivity to nonspecific adsorption of proteins. The reusability of sensor chip for detection of insulin was more than 25 cycles without an appreciable change in the original sensor activity.

In 2008, the study of protein immobilization on poly(pyrrole-co-pyrrole propylic acid) ( $\mathrm{PPy} / \mathrm{PPa}$ ) for immunosensing application was investigated by $\mathrm{Hu}$ et al. [26]. Goat IgG as a model protein was covalently immobilized on the carboxylcontaining of $\mathrm{PPy} / \mathrm{PPa}$ copolymer film through EDC/NHS as the coupling reagents. An electropolymerization process, protein covalent immobilization, and kinetics of antibodyantigen werein situ studied by SPR technique. Attenuated total reflection-Fourier transforms infrared (ATR-FTIR) and atomic force microscopy (AFM) were applied to characterize the prepared film. The evident peak around $1714 \mathrm{~cm}^{-1}$ on the ATR-FTIR spectra confirmed the existence of carboxyl groups in the prepared $\mathrm{PPy} / \mathrm{PPa}$ films. The maximum immobilization capability $\left(\sim 8 \mathrm{ng} / \mathrm{mm}^{2}\right)$ was achieved by an optimal monomer solution containing 10\% pyrrole propylic acid. The initial reaction between the antibody and antigen followed the first-order kinetics and the diffusion limitation was present during the detection. The SPR label-free detection of antigoat IgG demonstrated the power of SPR for in situ monitoring the polymer deposition, protein immobilization and sensing process, and the feasibility to fabricate a label-free SPR immunosensor.

The polypyrrole propylic acid (PPA) based on EC-SPR immunosensor was then reported by Dong et al. [12]. The polymer formation, probe immobilization, antigen-antibody interaction, and protein immunosensing process were in situmonitored by the EC-SPR measurement. The schematic diagram of in situ EC-SPR instrument for construction of immunosensor based on polypyrrole propylic acid (PPA) film is shown in Figure 1. PPA film was prepared by electropolymerization of pyrrole propylic acid monomer. Mouse IgG was used as a model analyte. Probe proteins were covalently immobilized with EDC/NHS as the coupling

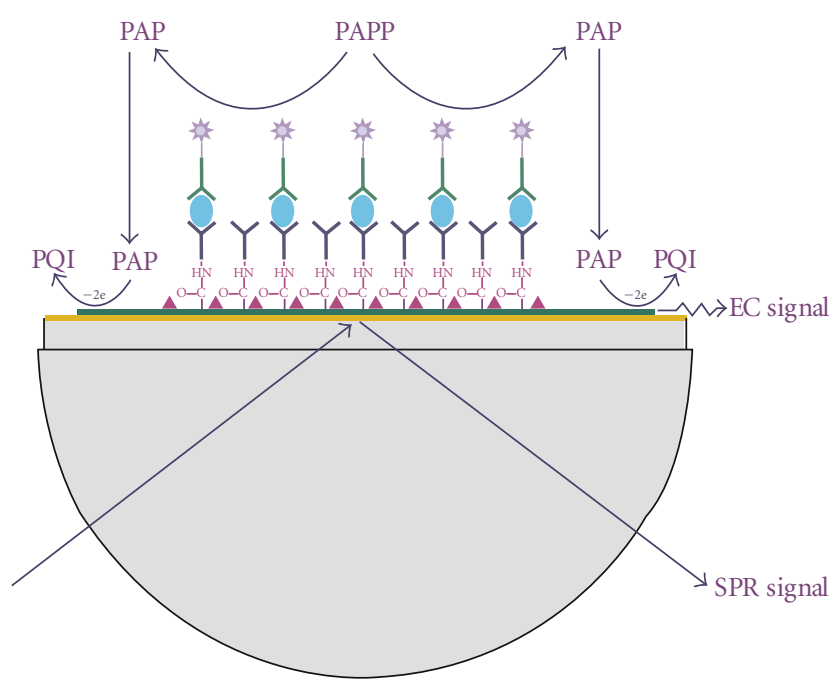

FIGURE 1: Schematic diagram of in situ EC-SPR instrument for construction of immunosensor based on polyporrole propylic acid (PPA) film [12].

reagents. The modified sensor chip was in situ investigated by SPR instrument with different concentrations of mouse IgG. The results showed that the introduction of captured antibody conjugated enzyme not only enhanced the current responses, but also increased the SPR angle shift. An approximate linear relationship could be obtained by plotting the data in semilogarithmic reference frame. The in situ ECSPR immunosensor described herein could have important potentials for diagnostics and medicine applications.

In 2010, the electrochemical controlled SPR immunosensor based on carboxylated polyaniline for the detection of human immunoglobulin G (IgG) without using further label molecule was reported by Sriwichai et al. [30]. Poly(3-aminobenzoic acid) (PABA) film was fabricated by electropolymerization of carboxylated aniline monomer (3aminobenzoic acid) on gold-coated high-reflective-index glass slide. The in situ EC-SPR was performed to study the kinetic property and electroactivity property of the polymer film. The SPR immunosensor-based PABA film was constructed by using monoclonal anti-human IgG produced by mouse as a model analyte. The immunosensor electrode was used to probe the binding reaction of antihuman/human IgG with various concentrations of human IgG at different constant applied potentials. The schematic diagram for construction of PABA-based immunosensor and sensing of human IgG is shown in Figure 2. The PABA film showed electroactivity in neutral solution. The SPR immunosensor based on PABA film could be used to detect human IgG in neutral solution at different constant applied potentials. Figure 3 shows SPR reflectivity response during construction of the immunosensor for sensing of $1 \mu \mathrm{g} / \mathrm{mL}$ human IgG at the constant applied potential of $0.4 \mathrm{~V}$. As shown in the figure, the reflectivity change was observed after the PABA film in phosphate-buffer saline (PBS) was activated by $N$-hydroxysuccinimide (NHS) and $\mathrm{N}$-(3dimethylaminopropyl)- $N$-ethylcarbodiimide hydrochloride 

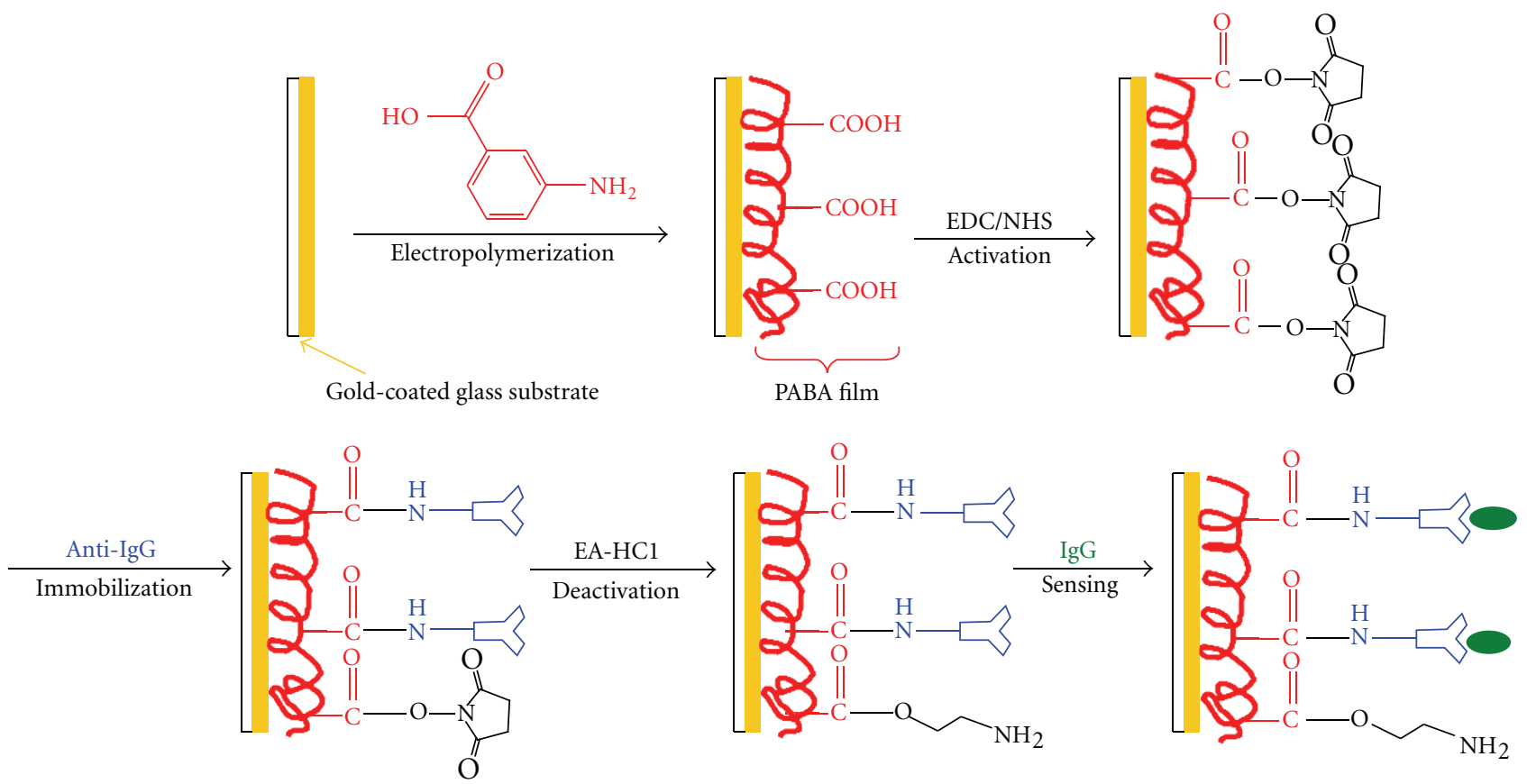

Figure 2: Schematic diagram of the construction of PABA-based immunosensor and sensing of human IgG [30].

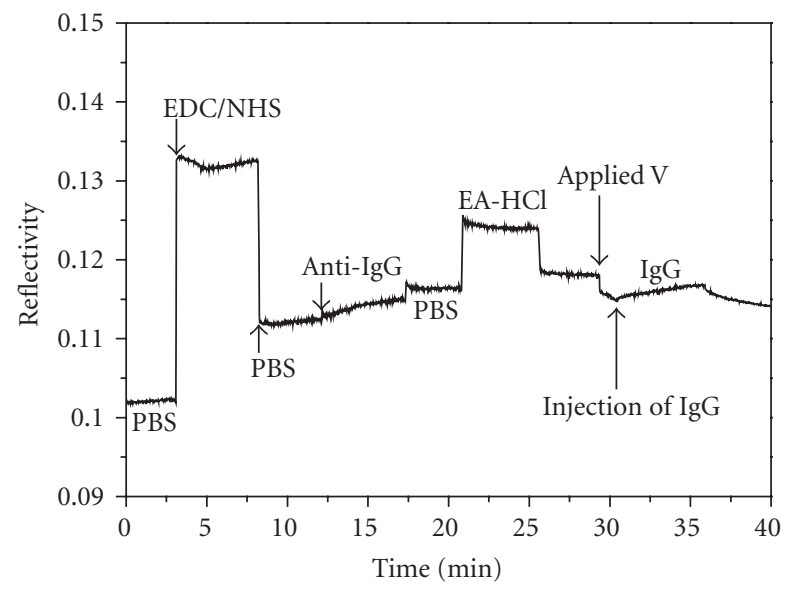

FIGURE 3: SPR response during construction of PABA-based immunosensor and sensing of human $\operatorname{IgG}(1 \mu \mathrm{g} / \mathrm{mL})$ at constant applied potential of $0.4 \mathrm{~V}[30]$.

(EDC). This process, called activation process, led to activation of carboxylic acid to NHS ester group. After rinsing with PBS, the anti-human IgG was then immobilized to the activated surface, which led to the formation of amide bond between NHS ester group and amine group of antihuman IgG. This process is called immobilization process. The reflectivity increased during both activation and immobilization processes. A buffer solution of ethanolaminehydrochloride $(\mathrm{EA}-\mathrm{HCl})$ was then employed to deactivate the residue of unreacted NHS ester group. After rinsing with PBS, constant potential was applied for 1 min before adding human IgG for sensing experiment. The SPR reflectivity increased during the sensing experiment, which is corresponding to the binding of anti-human IgG and human IgG. Different concentrations of human $\operatorname{IgG}(1,2.5,5$, $7,10 \mu \mathrm{g} / \mathrm{mL}$ ) were employed for sensing experiment at each constant applied potentials of open circuit, $0.4 \mathrm{~V}$ and $-0.2 \mathrm{~V}$ (results were not shown). The other results showed that higher binding amount of human IgG was obtained at applied potentials of -0.2 and $0.4 \mathrm{~V}$. The conducting polymer-based EC-SPR sensor could control the surface morphology and was a sensitive tool for enhancing the binding of biological molecules.

Recently, poly(pyrrole-3-carboxylic acid)- (PP3C-) based immunosensor investigated by in situ EC-SPR for monitoring the immobilization of anti-human IgG and its interaction with human IgG was reported by Janmanee et al. [39]. A pyrrole-3-carboxylic acid monomer was used for electropolymerization of PP3C film on gold surface. After the electropolymerization, SPR immunosensor was performed for the detection of human IgG, as a model analyte. The obtained PP3C-based immunosensor was employed for the detection of human IgG at various concentrations $(1-10 \mu \mathrm{g} / \mathrm{mL})$ with different constant potentials. The immunosensing process of the PP3C-based immunosensors was in situ monitored by EC-SPR measurement. The realtime SPR kinetic curve during the construction of PP3Cbased immunosensor shows the immobilization of antihuman IgG and the binding and dissociation process of anti-human IgG/human IgG. The SPR reflectivity change increased with applied constant potentials as shown in Figure 4(a), indicating that an electrochemically controlled PP3C film by applying the constant potential could be enhanced by the binding of human IgG to anti-human IgG on PP3C film, which is supported by AFM result. Moreover, 


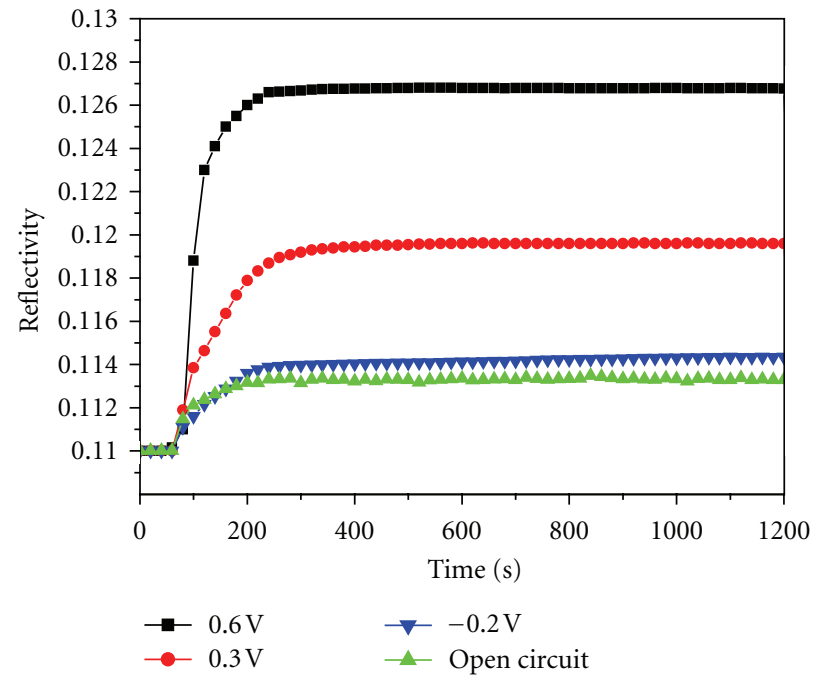

(a)

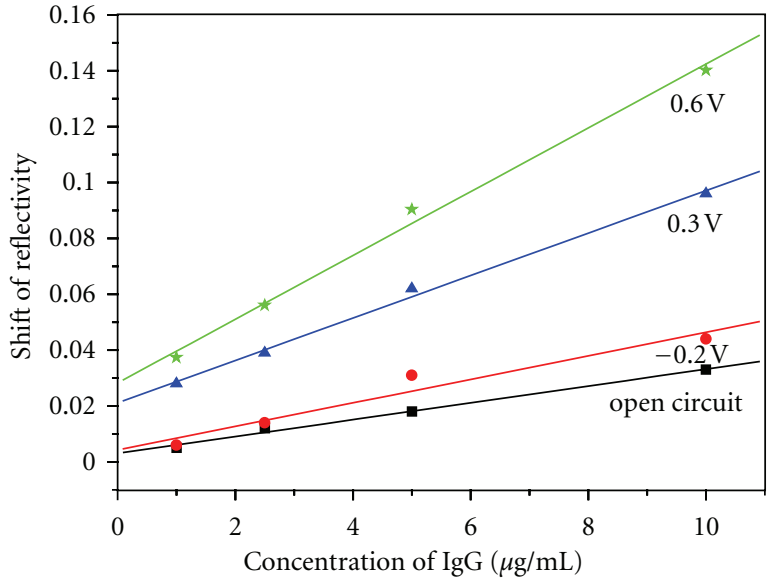

(b)

FIGURE 4: (a) SPR responses at different applied potentials for detection of human $\operatorname{IgG}(10 \mu \mathrm{g} / \mathrm{mL})$ at several constant potentials, and (b) the plot of the shift of reflectivity during binding process with concentration of IgG at different constant applied potentials [39].

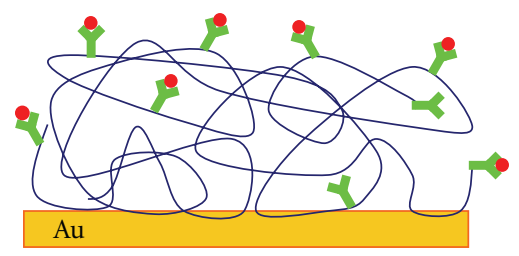

Anti-human IgG
- Human IgG

(a)

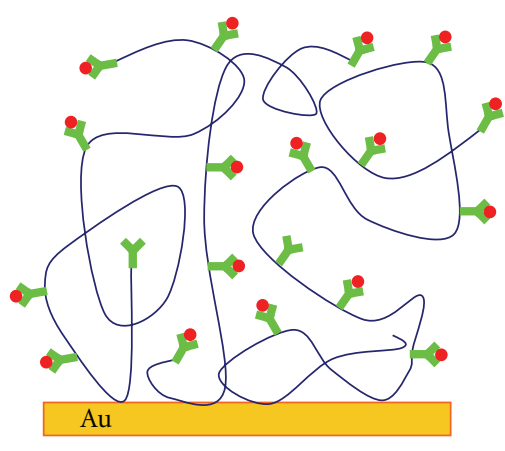

Anti-human IgG
Human IgG
PP3C

(b)

FIGURE 5: Schematic diagram of the binding process of anti-human IgG to human IgG on PP3C film (a) without applying potential and (b) with applying potential [39].

the binding amount of human IgG to anti-human IgG increased with increasing the concentration of human $\operatorname{IgG}$ as shown in Figure 4(b). The SPR reflectivity shifts show linear relationship with the concentrations of human IgG. The reflectivity change at applied potential of $0.6 \mathrm{~V}$, showing the highest sensitivity, was about 6-times more than that without applying potential. In addition, the sensitivity signal of the PP3C-based immunosensor shows a higher efficiency for the detection of anti-human IgG/human IgG compared with the standard MUA system. Figure 5 shows a schematic diagram of the binding process of anti-human IgG/human IgG on the PP3C film with applied potential and without applied potential. The surface morphology of PP3C film was changed with applied potential. The PP3C film swelled and the surface seemed to be rugged, leading to have more space for the binding of anti-human IgG and human IgG in the polymer chain, which leads to an increase of the sensitivity of immunosensor system.

\section{Acknowledgments}

The authors would like to acknowledge the financial support from the National Research University Project under Thailand's Office of the Higher Education Commission, Ministry of Education, and the "Global Circus" Program of Niigata University supported by the Ministry of Education, Culture, Sports, Science and Technology, Japan. The Graduate School, Chiang Mai University is also acknowledged. 


\section{References}

[1] N. K. Guimard, N. Gomez, and C. E. Schmidt, "Conducting polymers in biomedical engineering," Progress in Polymer Science, vol. 32, no. 8-9, pp. 876-921, 2007.

[2] A. Baba, S. Tian, F. Stefani et al., "Electropolymerization and doping/dedoping properties of polyaniline thin films as studied by electrochemical-surface plasmon spectroscopy and by the quartz crystal microbalance," Journal of Electroanalytical Chemistry, vol. 562, no. 1, pp. 95-103, 2004.

[3] J. C. Vidal, E. Garcia-Ruiz, and J. R. Castillo, "Recent advances in electropolymerized conducting polymers in amperometric biosensors," Microchimica Acta, vol. 143, no. 2-3, pp. 93-111, 2003.

[4] F. S. Damos, R. C. S. Luz, and L. T. Kubota, "Investigations of ultrathin polypyrrole films: formation and effects of doping/dedoping processes on its optical properties by electrochemical surface plasmon resonance (ESPR)," Electrochimica Acta, vol. 51, no. 7, pp. 1304-1312, 2006.

[5] B. Adhikari and S. Majumdar, "Polymers in sensor applications," Progress in Polymer Science, vol. 29, no. 7, pp. 699-766, 2004.

[6] Y. Wang and W. Knoll, "In situ electrochemical and surface plasmon resonance (SPR) studies of aniline-carboxylated aniline copolymers," Analytica Chimica Acta, vol. 558, no. 12, pp. 150-157, 2006.

[7] S. Toyama, K. Aoki, and S. Kato, "SPR observation of adsorption and desorption of water-soluble polymers on an Au surface," Sensors and Actuators, B, vol. 108, no. 1-2, pp. 903-909, 2005.

[8] M. Şenel and C. Nergiz, "Novel amperometric glucose biosensor based on covalent immobilization of glucose oxidase on poly(pyrrole propylic acid)/Au nanocomposite," Current Applied Physics, vol. 12, no. 4, pp. 1118-1124, 2012.

[9] M. Zhou and J. Heinze, "Electropolymerization of pyrrole and electrochemical study of polypyrrole: 1. Evidence for structural diversity of polypyrrole," Electrochimica Acta, vol. 44, no. 11, pp. 1733-1748, 1999.

[10] K. Shinbo, A. Baba, F. Kaneko et al., "In situ investigations on the preparations of layer-by-layer films containing azobenzene and applications for LC display devices," Materials Science and Engineering C, vol. 22, no. 2, pp. 319-325, 2002.

[11] M. Singh, P. K. Kathuroju, and N. Jampana, "Polypyrrole based amperometric glucose biosensors," Sensors and Actuators, B, vol. 143, no. 1, pp. 430-443, 2009.

[12] H. Dong, X. Cao, C. M. Li, and W. Hu, "An in situ electrochemical surface plasmon resonance immunosensor with polypyrrole propylic acid film: comparison between SPR and electrochemical responses from polymer formation to protein immunosensing," Biosensors and Bioelectronics, vol. 23, no. 7, pp. 1055-1062, 2008.

[13] J. J. Gooding, C. Wasiowych, D. Barnett, D. B. Hibbert, J. N. Barisci, and G. G. Wallace, "Electrochemical modulation of antigen-antibody binding," Biosensors and Bioelectronics, vol. 20, no. 2, pp. 260-268, 2004.

[14] J. W. Lee, F. Serna, J. Nickels, and C. E. Schmidt, "Carboxylic acid-functionalized conductive polypyrrole as a bioactive platform for cell adhesion," Biomacromolecules, vol. 7, no. 6, pp. 1692-1695, 2006.

[15] R. Schweiss, J. F. Lübben, D. Johannsmann, and W. Knoll, "Electropolymerization of ethylene dioxythiophene (EDOT) in micellar aqueous solutions studied by electrochemical quartz crystal microbalance and surface plasmon resonance," Electrochimica Acta, vol. 50, no. 14, pp. 2849-2856, 2005.
[16] R. D. Webster, "In situ electrochemical-ATR-FTIR spectroscopic studies on solution phase 2,4,6-tri-substituted phenoxyl radicals," Electrochemistry Communications, vol. 5, no. 1, pp. 6-11, 2003.

[17] R. D. Webster, R. A. W. Dryfe, J. C. Eklund, C. W. Lee, and R. G. Compton, "In situ electrochemical ESR studies of reactive radicals: the reductions of bromo-anthraquinone and methyl viologen," Journal of Electroanalytical Chemistry, vol. 402, no. 1-2, pp. 167-174, 1996.

[18] R. Kurita, K. Nakamoto, A. Ueda, and O. Niwa, "Comparison of electrochemical and surface plasmon resonance immunosensor responses on single thin film," Electroanalysis, vol. 20, no. 20, pp. 2241-2246, 2008.

[19] R. Kurita, Y. Yokota, A. Ueda, and O. Niwa, "Electrochemical surface plasmon resonance measurement in a microliter volume flow cell for evaluating the affinity and catalytic activity of biomolecules," Analytical Chemistry, vol. 79, no. 24, pp. 9572-9576, 2007.

[20] W. Knoll, "Interfaces and thin films as seen by bound electromagnetic waves," Annual Review of Physical Chemistry, vol. 49, no. 1, pp. 569-638, 1998.

[21] A. Baba and W. Knoll, "Properties of poly(3,4-ethylenedioxythiophene) ultrathin films detected by in situ electrochemicalsurface plasmon field-enhanced photoluminescence spectroscopy," The Journal of Physical Chemistry B, vol. 107, no. 31, pp. 7733-7738, 2003.

[22] K. Kato, K. Yamashita, Y. Ohdaira, A. Baba, K. Shinbo, and F. Kaneko, "Electrochemical surface plasmon excitation and emission light properties in poly(3-hexylthiophene) thin films," Thin Solid Films, vol. 518, no. 2, pp. 758-761, 2009.

[23] S. Tian, A. Baba, J. Liu et al., "Electroactivity of polyaniline multilayer films in neutral solution and their electrocatalyzed oxidation of $\beta$-nicotinamide adenine dinucleotide," Advanced Functional Materials, vol. 13, no. 6, pp. 473-479, 2003.

[24] J. C. C. Yu and E. P. C. Lai, "Polypyrrole film on miniaturized surface plasmon resonance sensor for ochratoxin A detection," Synthetic Metals, vol. 143, no. 3, pp. 253-258, 2004.

[25] Y. Shao, Y. Jin, J. Wang, L. Wang, F. Zhao, and S. Dong, "Conducting polymer polypyrrole supported bilayer lipid membranes," Biosensors and Bioelectronics, vol. 20, no. 7, pp. 13731379, 2005.

[26] W. Hu, C. M. Li, and H. Dong, "Poly(pyrrole-co-pyrrole propylic acid) film and its application in label-free surface plasmon resonance immunosensors," Analytica Chimica Acta, vol. 630, no. 1, pp. 67-74, 2008.

[27] J. Wang, F. Wang, H. Chen, X. Liu, and S. Dong, "Electrochemical surface plasmon resonance detection of enzymatic reaction in bilayer lipid membranes," Talanta, vol. 75 , no. 3 , pp. 666-670, 2008.

[28] X. Kang, G. Cheng, and S. Dong, "A novel electrochemical SPR biosensor," Electrochemistry Communications, vol. 3, no. 9, pp. 489-493, 2001.

[29] K. V. Gobi, H. Iwasaka, and N. Miura, "Self-assembled PEG monolayer based SPR immunosensor for label-free detection of insulin," Biosensors and Bioelectronics, vol. 22, no. 7, pp. 1382-1389, 2007.

[30] S. Sriwichai, A. Baba, S. Phanichphant, K. Shinbo, K. Kato, and F. Kaneko, "Electrochemically controlled surface plasmon resonance immunosensor for the detection of human immunoglobulin G on poly(3-aminobenzoic acid) ultrathin films," Sensors and Actuators, B, vol. 147, no. 1, pp. 322-329, 2010 . 
[31] H. Peng, L. Zhang, C. Soeller, and J. Travas-Sejdic, "Conducting polymers for electrochemical DNA sensing," Biomaterials, vol. 30, no. 11, pp. 2132-2148, 2009.

[32] K. S. V. Santhanam, "Conducting polymers for biosensors: rationale based on models," Pure and Applied Chemistry, vol. 70, no. 6, pp. 1259-1262, 1998.

[33] M. Yuqing, C. Jianrong, and W. Xiaohua, "Construction of a glucose biosensor by immobilizing glucose oxidase within a poly(o-phenylenediamine) covered screen-printed electrode," Journal of Biological Sciences, vol. 6, no. 1, pp. 18-22, 2006.

[34] D. D. Ateh, H. A. Navsaria, and P. Vadgama, "Polypyrrolebased conducting polymers and interactions with biological tissues," Journal of the Royal Society Interface, vol. 3, no. 11, pp. 741-752, 2006.

[35] 2010, http://www.immunosensors.com/.

[36] 2010, http://www.mdpi.com/journal/sensors/special_issues/ immunosensors.

[37] A. Ramanavicius, A. Finkelsteinas, H. Cesiulis, and A. Ramanaviciene, "Electrochemical impedance spectroscopy of polypyrrole based electrochemical immunosensor," Bioelectrochemistry, vol. 79, no. 1, pp. 11-16, 2010.

[38] J. Wang, F. Wang, Z. Xu, Y. Wang, and S. Dong, "Surface plasmon resonance and electrochemistry characterization of layer-by-layer self-assembled DNA and $\mathrm{Zr}^{4+}$ thin films, and their interaction with cytochrome c," Talanta, vol. 74, no. 1, pp. 104-109, 2007.

[39] R. Janmanee, A. Baba, S. Phanichphant et al., "Detection of human IgG on Poly(pyrrole-3-carboxylic acid) thin film by electrochemical-Surface plasmon resonance spectroscopy," Japanese Journal of Applied Physics, vol. 50, no. 1, Article ID 01BK02, 6 pages, 2011. 

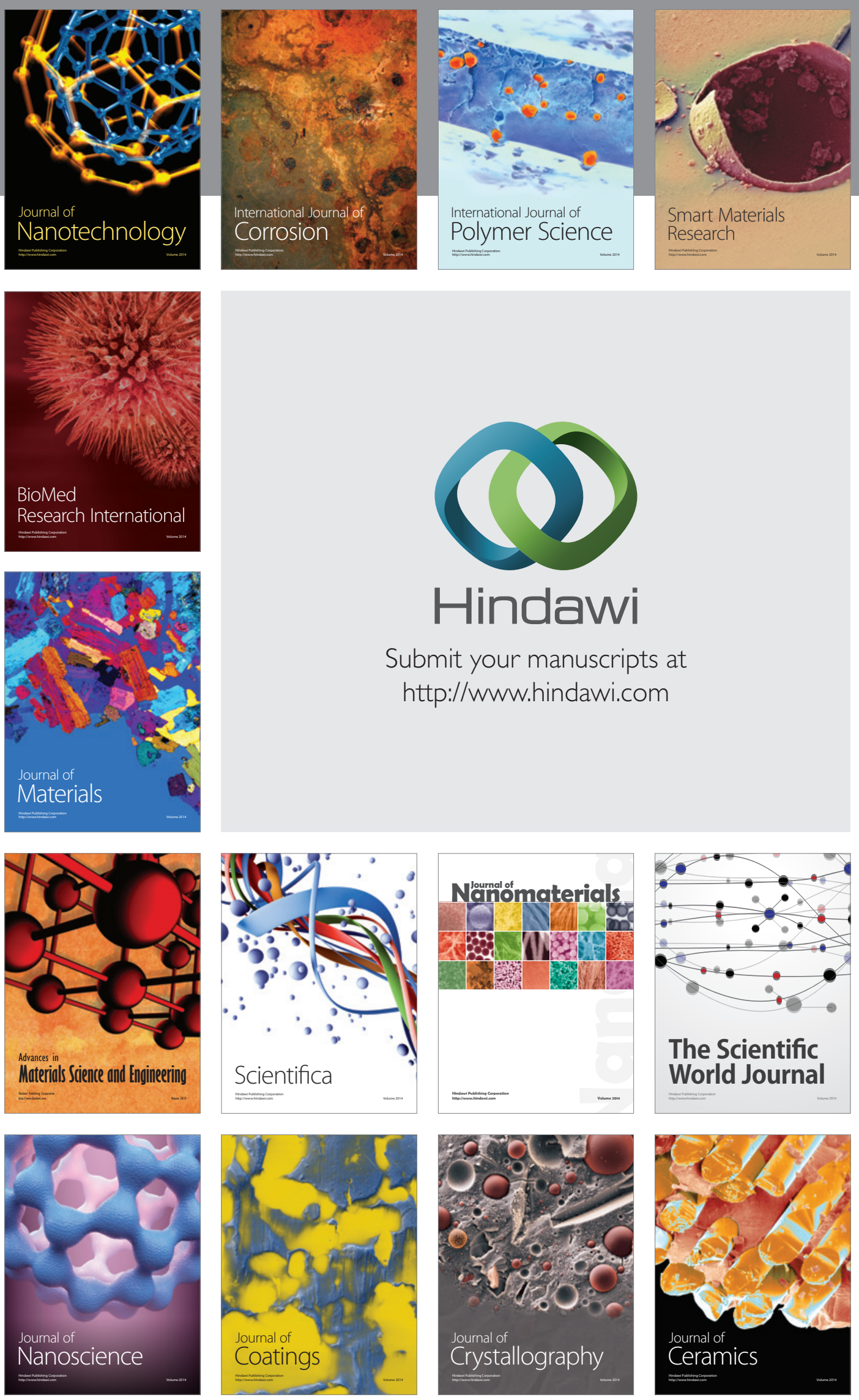

The Scientific World Journal

Submit your manuscripts at

http://www.hindawi.com

\section{World Journal}

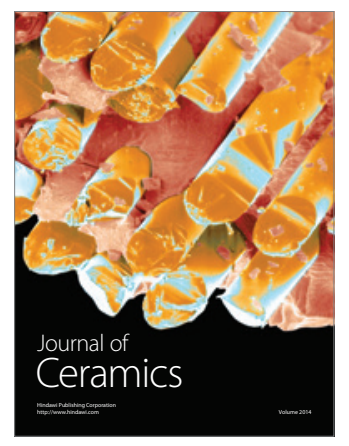

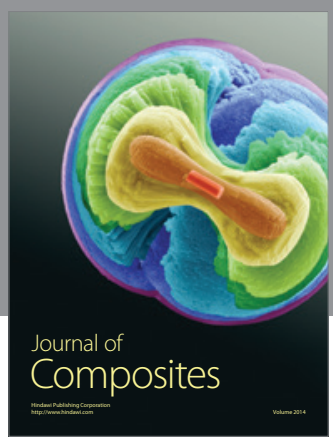
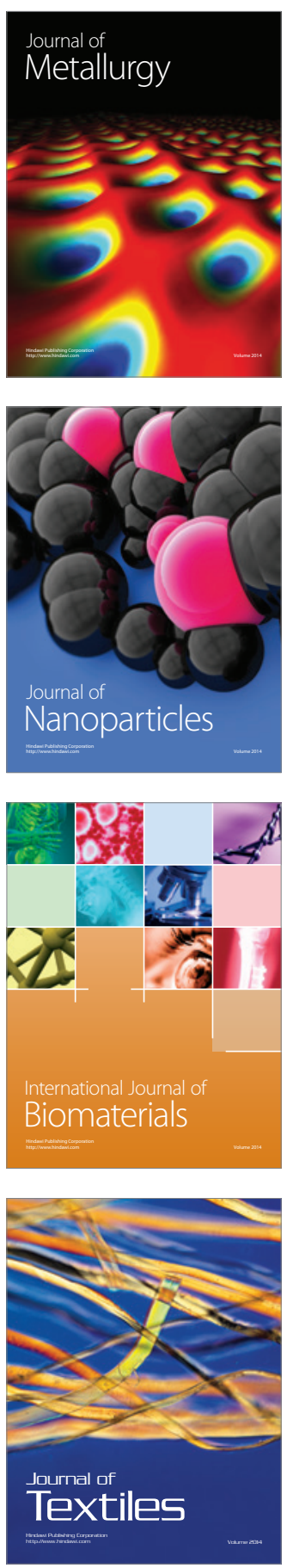